\title{
Leber's hereditary optic neuropathy, intellectual disability and epilepsy presenting with variable penetrance associated to the m.3460G $>$ A mutation and a heteroplasmic expansion of the microsatellite in MTRNR1 gene - case report
}

Angelica Bianco ${ }^{1}$, Luigi Bisceglia², Maria Fara De Caro', Valeria Galeandro' ${ }^{1}$ Patrizia De Bonis ${ }^{2}$, Apollonia Tullo ${ }^{3}$, Stefano Zoccolella ${ }^{1}$, Silvana Guerriero ${ }^{1}$ and Vittoria Petruzzella ${ }^{1 *}$ (i)

\begin{abstract}
Background: Leber's hereditary optic neuropathy (LHON) associated with mutations in mitochondrial DNA (mtDNA) typically manifests only optic nerve involvement but in some patients may develop additional neurological complications. The cause of this association is not clear.

Case presentation: We present a case of a 24-year-old male with a history of subacute, painless, and rapidly progressive bilateral vision loss. We performed ophthalmological, neurological and neuropsychological investigations in the proband and his LHON family. The proband showed optic neuropathy, epilepsy, migraine, and intellectual disability; all the maternal relatives did not manifest optic neuropathy but a moderate to severe intellectual disability. Genetic screening revealed a novel association of the LHON m.3460G > A primary mutation with the m.T961deIT $+C(n)$ ins within the mitochondrial encoded 12S RNA (MTRNR1) gene which segregates with the intellectual disability through the maternal branch of the family. We also found a significant increase of mtDNA content in all the unaffected homo/heteroplasmic mutation carriers with respect to either affected or control subjects.
\end{abstract}

Conclusion: This is the first case reporting the co-segregation of a mutation in MTRNR1 gene with a LHON primary mutation, which may be a risk factor of the extraocular signs complicating LHON phenotype. In addition, the data herein reported, confirmed that the key factor modulating the penetrance of optic atrophy in the family is the amount of mtDNA.

Keywords: LHON, Penetrance, Intellectual disability, Mitochondrial DNA, MTRNR, M.3460G > A

\footnotetext{
* Correspondence: vittoria.petruzzella@uniba.it

'Dipartimento di Scienze Mediche di Base, Neuroscienze e Organi di Senso,

Università degli Studi Aldo Moro, Piazza G. Cesare, 70124 Bari, Italy

Full list of author information is available at the end of the article
}

(c) The Author(s). 2018 Open Access This article is distributed under the terms of the Creative Commons Attribution 4.0 International License (http://creativecommons.org/licenses/by/4.0/), which permits unrestricted use, distribution, and reproduction in any medium, provided you give appropriate credit to the original author(s) and the source, provide a link to the Creative Commons license, and indicate if changes were made. The Creative Commons Public Domain Dedication waiver (http://creativecommons.org/publicdomain/zero/1.0/) applies to the data made available in this article, unless otherwise stated. 


\section{Background}

Leber's Hereditary Optic Neuropathy (LHON), is due to three primary mutations (i.e. m.3460G $>\mathrm{A}$ in $M T-N D 1$, m.11778G > A in MT-ND4 and m.14484 T > C MT-ND6) in the mitochondrial DNA (mtDNA) genes, encoding for three different subunits of NADH: ubiquinone oxidoreductase (EC 1.6.5.3) or complex I (CI) for more than $90 \%$ of cases [1]. LHON is typically characterized by a rapid bilateral central vision loss owing to focal degeneration of the retinal ganglion cell layer and optic nerve $[2,3]$. Typically, not all the individuals who inherit LHON primary mutations develop optic neuropathy and visual impairment [4] thus accounting for the extremely variability of penetrance in families. In some cases, the ophthalmological signs may be complicated by additional neurological signs such as dystonia [5-8], parkinsonism $[5]$, cerebellar ataxia $[9,10]$, epilepsy $[11,12]$, myoclonus $[13,14]$, juvenile-onset encephalopathy and psychiatric disturbances [1]. Neither the highly variable penetrance of the optic neuropathy - which is always much greater in males - or the presence of extraocular signs can be exclusively explained by the presence of primary mutations that are necessary but not sufficient to cause the disease. All these elements suggest that other genetic and/or environmental factors must influence the phenotype. From a genetic standpoint, the mtDNA itself may contribute in various ways to LHON manifestation: the homo- or heteroplasmy conditions of the primary mutation $[15,16]$; the presence of additional mtDNA mutations [17]; the number of mtDNA copies within the cells [18-21].

Herein, we assessed the presence and the homo/heteroplasmic status of the m.3460G > A LHON mutation; the co-occurrence with novel mutation m.T961delT + insC(n) within the mitochondrial encoded 12S RNA (MTRNR1) gene and the mtDNA cellular content in a family manifesting ocular neuropathy complicated by intellectual disability, migraine and epilepsy as non-ophthalmologic features.

\section{Case presentation}

The proband, a 24 year old male from Southern Italy, presented at age 16ys to the Ophthalmology Clinic, Policlinico Hospital, Italy, with a history of subacute, painless, and rapidly progressive bilateral vision loss. At the time of presentation, the proband appeared to be healthy, a well-developed boy but with clear signs of anxiety. One month prior to presentation, he had noticed impaired sight at his right eye and, within a few days, he could only see shadows. One week after the loss of sight in his right eye, the same symptom occurred in his left eye. Ophthalmologic examinations, at the moment of hospitalization, revealed at right eye (RE), BCVA of 20/200; hyperemic optic disk, tortuosity, and telangiectasia of retinal vasculature and absence of leakage and staining of the retinal vessels were revealed using fluorescein angiography. OCT examination showed an increase of nerve fibers layer thickness (average RNFL 108.58) and CVC examination revealed a deep central scotoma at RE, while there was not any alteration at left eye (LE). After six weeks, the young man presented the same symptomatology at his LE. Progressively, both optic nerves developed atrophy that was documented by OCT examination. CVC examination was no more possible because the visual acuity dropped to only light perception. All the family members underwent a complete ophthalmological examination. Visual acuity was 20/20 in all members. Fundus examination showed a hyperemic optic disk and vessels tortuosity in the proband's young brother (III:2) and sister (III:3), whereas it was normal for the other family members. Visual field analysis was performed in all the available family members (Fig. 1) and resulted within normal ranges as well as the OCT-RNFL thickness examinations that were also in the normal range (NR: 97.3-/+9.6 $\mu \mathrm{m}$ ). After disease onset, the proband started ubiquinone analog therapy with Idebenone (100 mg b.i.d.) without any improvement of visual acuity. Additionally, the proband had experienced epilepsy at 13 years of age, with recurrent tonic-clonic seizures that were under control with Oxcabazepine tablets $1800 \mathrm{mg} /$ day, Topiramate tablets $400 \mathrm{mg} /$ day, paroxetine hydrochloride tablets $20 \mathrm{mg} /$ day, Pregabalin tablets $450 \mathrm{mg} /$ day, Lorazepam tablets $1 \mathrm{mg} /$ day as needed. He had no history of smoking, alcohol and of any illicit drug use. Interestingly, family history was significant for intellectual disability and hyperactivity in the mother (II:1) and siblings (III:2 and III:3) (Fig. 1) as outlined below; no one of the family used tobacco or alcohol with the exception of II:3 who referred tobacco abuse (Fig. 1).

To measure intelligence as IQ score in agreement with the age of patients, the Wechsler Intelligence Scalefor Children - Revised (WISC-R) was used for subjects aged 6 to 16 years, whereas the Wechsler Adult Intelligence Scale - Revised (WAIS-R) for subjects aged 17 to 90 years. The test showed that the proband manifested mild intellectual disability, whereas his available relatives all resulted in borderline intellectual functioning (Table 1). Recently, the youngest brother (III-2), who was suffering from severe migraine, at the age of 20ys, has developed recurrent seizures such as the proband, that are under control by Clonazepam $2.5 \mathrm{mg}$ drops (12 drops/day) and Lacosamide tablets $400 \mathrm{mg} /$ day. This study was conducted with the approval of the Institutional Review Board of Azienda Consorziale Policlinico Bari and Bari University. Before recruitment into our study, all participants and their guardians signed written informed consent in accordance with the guidelines of the Declaration of Helsinki. This research abided by the process of collection of data from patients with genetic diseases and the requirements of the Italian Ministry of Public Health. Written informed consent was 


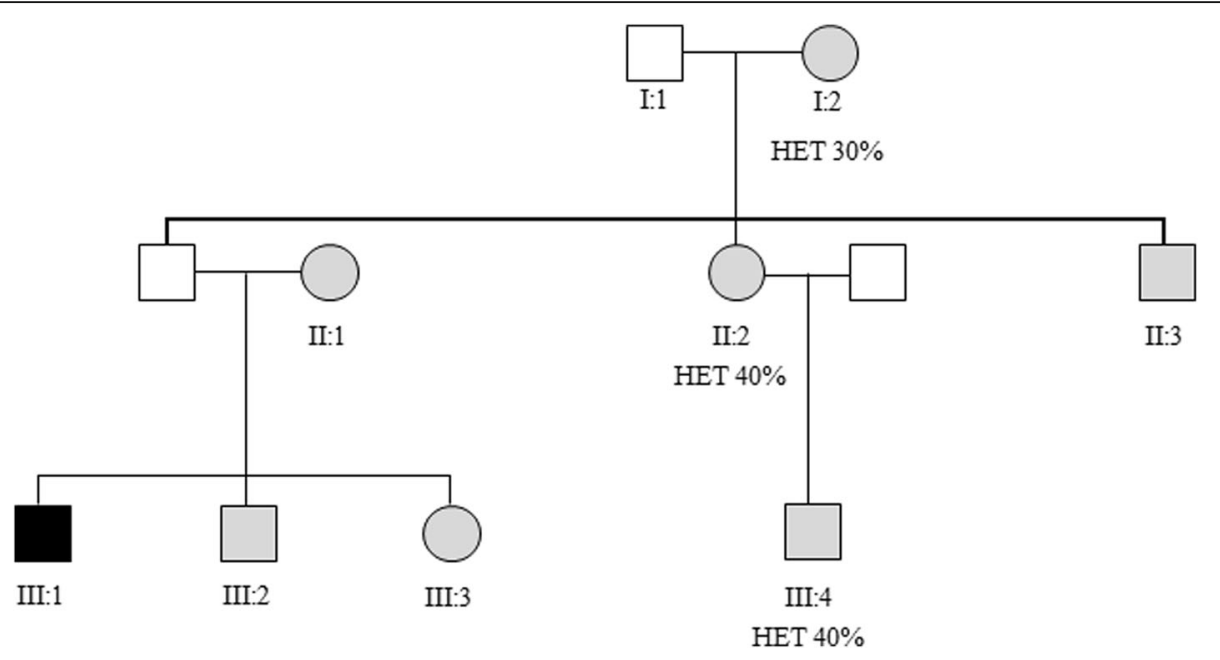

Fig. 1 Pedigree of the m.3460G > A family. The black symbol indicates the proband; gray symbols indicate asymptomatic relatives. Percentages of heteroplasmy of m.3460G > A mutation are reported

obtained from the participant or from their parents for publication of this Case report.

Total genomic DNA was extracted by standard methods from peripheral blood of the patient and his relatives and from control subjects. Mitochondrial DNA genetic analysis was positive for the LHON m.3460G > A primary mutation in the proband and in all the family members; the mutation resulted as homoplasmic in the proband as well as in four LHON unaffected (II-1; II-3; III-2; III-3) while it was heteroplasmic in three LHON unaffected (I-2; II-2; III-4) subjects Table 1; showing a mean frequency of the mutant allele of $35 \%$ (range 3040\%) (Fig. 1).

We then measured mtDNA copy number, estimated as mtDNA/nDNA ratio [22], in peripheral blood samples from the proband and unaffected family members and then they were compared to control group's (Table 1). The control group consisted of 90 unrelated subjects who had no history of a retinal disease, eye trauma or surgery, nor any evidence of systemic or neurological disease. MtDNA copy number of the homoplasmic relatives was evaluated in previous work [19]. Frequency distribution of the mtDNA copy number showed that the peak of mtDNA content shifted progressively towards higher values from control $(210 \pm 86)$ to affected $(240 \pm 86)$ to unaffected $(548 \pm 217)$ subjects with very high statistical significance: controls versus unaffected subjects, $P<0.001$; proband versus unaffected subjects, $P<0.001$ (ANOVA test). Furthermore, though the limitation of the sample size, when we compared mtDNA copy numbers between subjects harboring homo- or heteroplasmic m.3460G > A mutation, no difference was observed.

We considered the peculiarity of the clinical presentation of the proband, so we reasoned that additional mutations might contribute to the phenotype; we performed
Sanger sequencing of the entire mtDNA genome [23]. All nucleotide variants were annotated according to the procedure described in MtoolBox [24] and are reported in Additional file 1. We identified 55 variants of which 46 contributed to defining the haplogroup U4a1a and 19 variants were prioritized (Additional file 1). Interestingly, the proband showed multiple species of mtDNA molecules of variable lengths due to the variability of the number of cytosines inserted in the microsatellite at position m.961 of the MTRNR1 gene which normally contains a $(C) 5 \mathrm{~T}(\mathrm{C}) 4$ poly-cytidine tract (NC_012920.1; www.ncbi.nlm.nih.gov). We performed Sanger sequencing of MTRNR1 region in all available relatives, confirming also in these, the microsatellite instability. The specific m.961delT $+\mathrm{C}(\mathrm{n})$ ins variant is annotated in Mitomap database (www.mitomap.org) mostly associated to deafness, but no population data are available for it and for variants harboring insertion with a number of $\mathrm{C}$ more than 7 (dbSNP link: https://www.ncbi.nlm.nih.gov/variation/ view $/$ ?chr $=$ MT \&from $=961 \&$ to=961\&mk=961\%3A961\%7 CNC_012920.1\&assm=GCF_000001405.25). To discriminate among the different lengths of molecules carrying $\mathrm{C}(\mathrm{n})$-microsatellite (mtMS) we cloned the proband appropriate DNA regions and then picked different clones for direct sequencing. We found eight additional species carrying the insertion of 1 to $8 \mathrm{Cs}$ starting at position m.961 of the wild-type molecule, which corresponds to a microsatellite of 10 to $17(\mathrm{C})$. To better investigate on the mtMS instability (mtMSI), fragment analysis was performed in all the available family members. This analysis showed that the MTRNR1 mtMSs may have different lengths and that is heteroplasmic with variable percentages (Fig. 2; Additional file 2). I-2 had from 9 to 13(C); II-1, II-2, and II-3 had from 9 to $14(\mathrm{C})$ and 15(C), respectively; III-1 (proband), as well as III-2, III-3 and III-4 had 


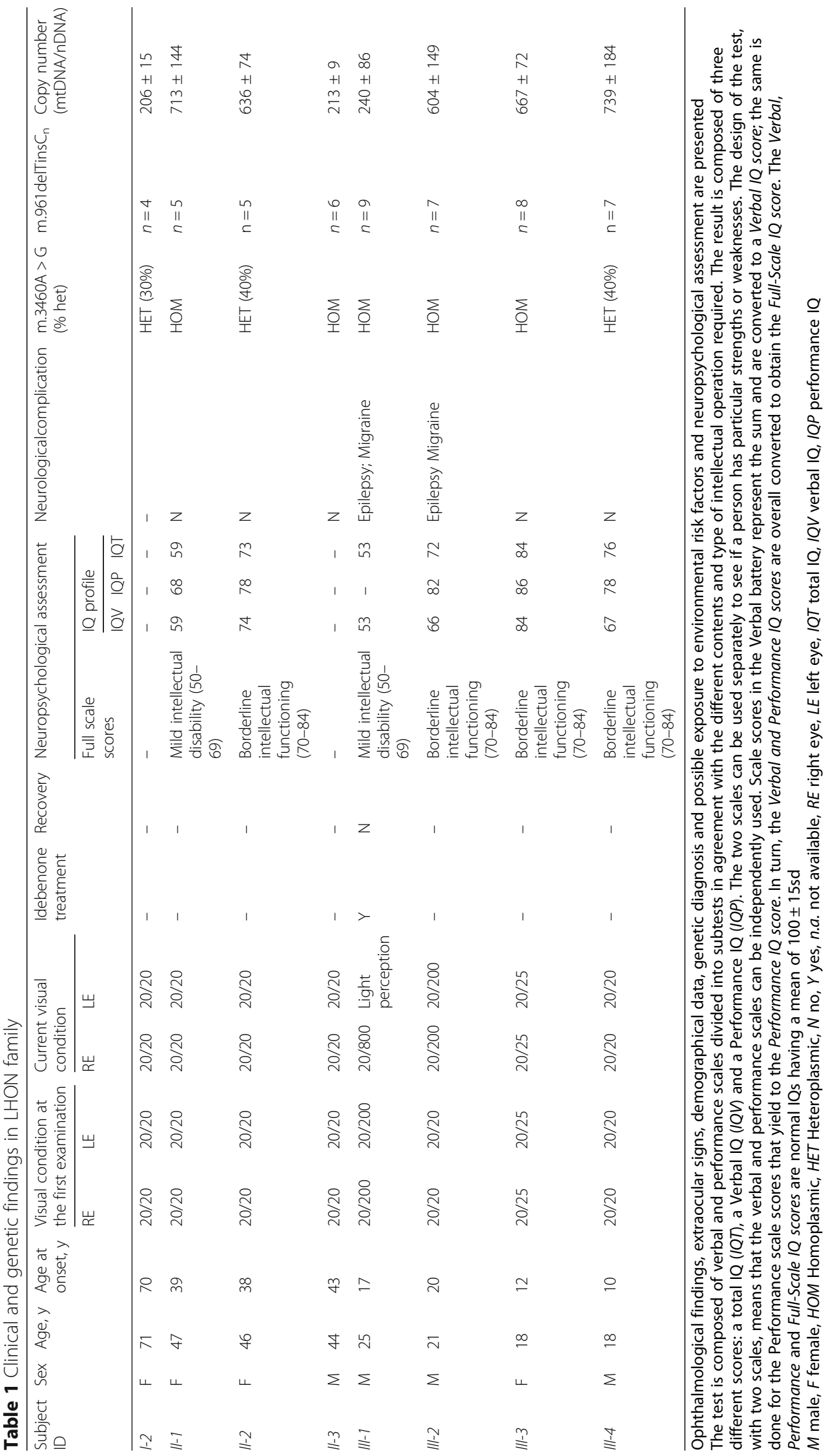



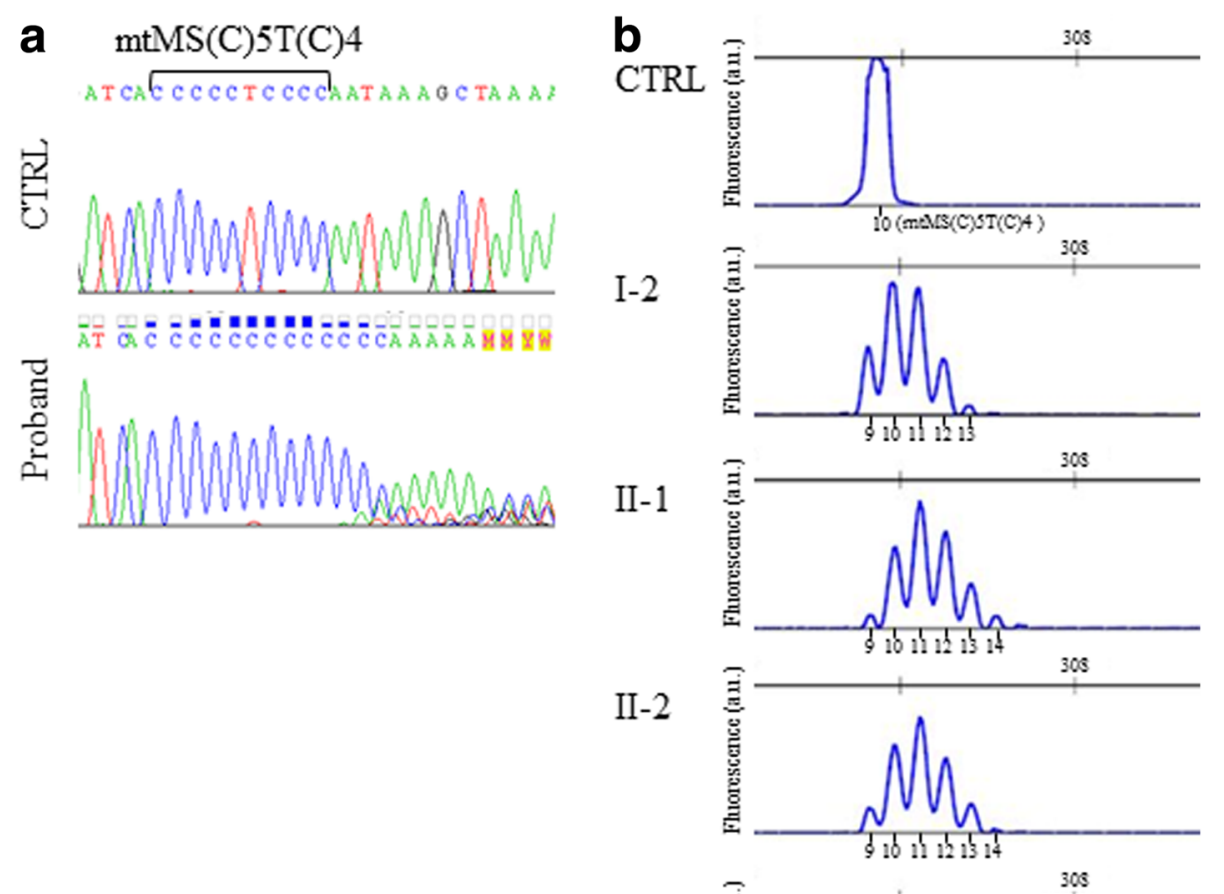

II-3

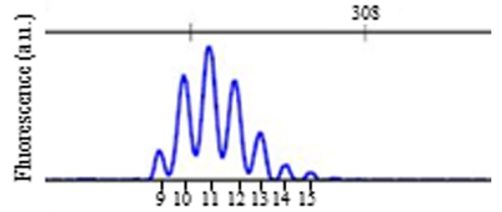

III-1

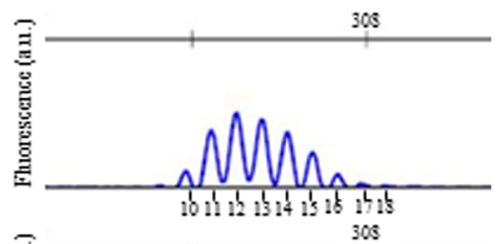

III-2

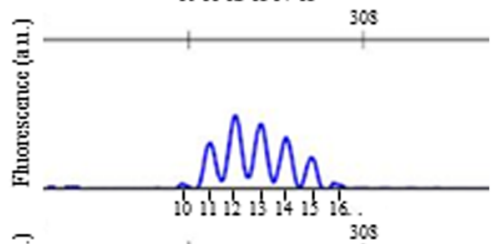

III-3

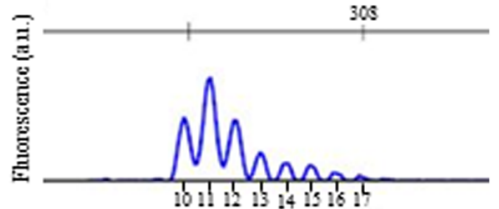

III-4

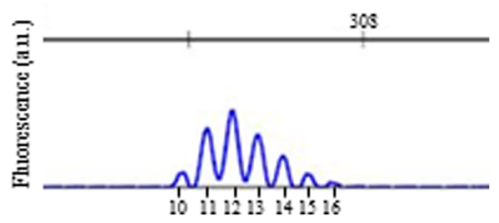

Fig. 2 Analysis of the C-microsatellite in LHON family. a Sanger sequencing of the mtMS region of the proband and control. $\mathbf{b}$ Fragment analysis of mtMS in all the maternal family members

from 9 to 16 (C); III-1 and III-3 showed also additional peaks i.e. $17(\mathrm{C})$ and $18(\mathrm{C})$. Quantitative analysis considering both the area and the height of the peaks showed that the $10(\mathrm{C})$ microsatellite was the most represented species in $\mathrm{I}: 2$; in the second generation, there is a prevalence of $10-12(\mathrm{C})$ species, whereas in the third generation, there is 
a decrease of 10-12(C) species amount, with the exception of III-3, and a surge of $13(\mathrm{C})$ and especially of 14$16(\mathrm{C})$ and even of 17 and $18(\mathrm{C})$ which, on the contrary, were not at all present in the second generation (Additional file 2). In order to investigate the possible functional effect of the C-microsatellite expansion, the prediction of MTRNR1 secondary structure and folding performed by RNA fold software from Vienna RNA package [25], assessed that m.961delT $+\mathrm{C}(\mathrm{n})$ ins would expand the size of a proposed loop structure in the MTRNR1.

\section{Discussion and conclusions}

We identified an LHON pedigree characterized by intellectual disability, migraine, and epilepsy as extra-ocular signs which harbor a novel association of the m.3460G > A mutation with the instability of the C-microsatellite in the MTRNR1 gene. In the LHON family, the m.3460G > A primary mutation was homoplasmic in the proband and either homo- or heteroplasmic in the seven LHON unaffected relatives. The heteroplasmic LHON unaffected subjects showed a mean percentage of $35 \%$ of mutated respect to total mtDNA, which is far below the previously reported $60 \%$ threshold predicted to represent the risk of visual loss [15]. Such evidence might be compatible with the notion true for the vast majority of mitochondrial diseases that the presence of wild-type in coexistence with mutated mtDNA might protect from the vision injury [15]. Nevertheless, the identification in four unaffected relatives (II-1; II-3; III-2; III-3) of homoplasmic LHON mutation annulled the hypothesis that the presence of wild-type together with m.3460G > A mutant alleles could be protective for visual loss. This suggests that additional protective factors must be considered. Furthermore, we found that the proportion of the m.3460G > A mutant allele does not increase in the successive generations (I-1: 30\%; II-2: 40\%; III-4: 40\%) suggesting a random genetic drift mechanism behind the transmission of the heteroplasmic LHON mutation [26].

To the aim of investigating on further protective/risk factors for explaining the variability of LHON penetrance, we evaluated mtDNA content in peripheral blood cells in all the family members. We found a significant and consistent increase of mtDNA in unaffected subjects respect to the proband and controls which was independent of the homo/heteroplasmic status of the m.3460G > A mutation. Recently, comparison of mtDNA copy number from peripheral blood cells of unaffected, affected, and control subjects have shown that unaffected subjects have a significant increase of mitochondrial content per cell suggesting that it may be protective by a compensatory response to respiratory chain dysfunction [18-21]. Indeed, we found two exceptions to this general 'rule': the grandmother's (I-2; 70ys) copy number was as low as the subject (II-3) and similar to normal controls'. Indeed, this result agrees with the knowledge that aging [22] as well as low estrogen condition, such that of a 70 yr-old woman [18, 19], are conditions known to determine a decrease in mtDNA content. The second case referred tobacco abuse; smoke has been shown to reduce mtDNA copy number in all cell types [27]. The evaluation of copy number in the peripheral blood was performed on samples obtained at first neurological exam of the family, before that the youngest brother (III-2) of the proband, developed decline of sight, migraine and seizures at the age of 20ys.

In the present family, LHON is accompanied by additional neurological and psychiatric manifestations, i.e. migraine, epilepsy and intellectual disability. Intellectual disability was present in all the maternal relatives. Formerly, in the original paper by Kwittken et al. 1958 some LHON patients were reported affected also by headache, vertigo, epilepsy, intellectual impairment, nystagmus, tremor, areflexia, loss of sphincter control, pyramidal tract disease, ataxia, or sensory disturbances among the LHON-associated extraocular signs [28]. Later, additional reports have provided evidence that LHON may manifest not exclusively as an ophthalmologic disorder but rather as a multisystemic disease with a predominant affection of the eyes $[29,30]$. The genetic bases of the multisystem involvement had not been investigated thoroughly.

Intellectual disability, migraine, and epilepsy as extra-ocular signs were found in the herein described LHON pedigree of three generations. The proband and all the maternal relatives have, in addition to the m.3460G > A mutation, a heteroplasmic expansion of the poly-cytidine tract of the microsatellite at $\mathrm{m} .961 \mathrm{in}$ MTRNR1gene. The m.961 C-stretch represents one of the overall $14 \mathrm{mtMSs}$ found in the D-loop and in different coding regions of the mtDNA. This region is not evolutionarily strictly conserved among mammals but it is highly specific of Homo sapiens thus pointing to a functional restriction specific for humans [31]. Interestingly, it has never been associated with pathological conditions including intellectual disability. In the family, we found that the relative proportions of the $\mathrm{C}$-microsatellite region lengths are maintained and expanded in maternally relatives suggesting that the first event of expansion had arisen in the grandmother - or possibly even before - and then had reached fixation within individuals as a consequence of misalignment and/or slippage during replication when stuttering can generate mixed population of C-mtMSs having different lengths. During the following generations, the transmission of the shortest mtMSs together with the expansion of mtMSs length may take place which generates heteroplasmy of the mtMS [32]. It has been proposed that $\mathrm{m} .961$ position per se, either as transition or deletion in the middle of the $\mathrm{C}$-microsatellite, may affect the tertiary or quaternary structure of the small 
ribosomal subunit thus impairing protein synthesis [33]. Moreover, once the G-rich region is transcribed in RNA, it may form a strong G-quadruplex structure leading to termination of RNA synthesis, as it occurs during primer formation for mtDNA replication [34]. If this mechanism takes place, we can hypothesize a reduction in rRNA steady-state level and ribosome assembly. But both hypotheses cannot be tested because we do not have cells deriving from the family members.

It is widely reported that MTRNR1 gene is a hotspot for mutations associated with aminoglycoside ototoxicity [35-40]. The variable C-cluster of the MTRNR1 region maps between 21 and 22 loops of the secondary structure of the ribosomal RNA [41] and has been implicated as having a role in the phenotypic expression of the m.1555A > G mutation in a large Chinese pedigree [42]. Deafness or hearing loss was not described among signs either in the proband or in his relatives but we cannot exclude that up to now no one had ever been exposed to aminoglycosides. Typically, intellectual disability may be part of a more complex syndromic condition and, to our knowledge, it has never been associated with mtDNA alterations. Though we cannot exclude that there might be a coincidence of an LHON mutation with a mutation in nuclear genes associated with intellectual disability [43] and epilepsy [44], we noticed that the neurologic and psychiatric signs manifested as a strong maternal inherited trait. The peculiar pattern of MTRNR1 mutation as well as the presence of the longest species of mtMS segregating with intellectual disability is suggestive of their association although the size of the family does not allow to make a statistically significant link between the presence of the $961 \mathrm{Tdel}+\mathrm{C}(\mathrm{n})$ ins variant and the non-LHON clinical features detected in the family. Therefore, the possible synergistic effect of the 961Tdel $+\mathrm{C}(\mathrm{n})$ ins with the $3460 \mathrm{G}>\mathrm{A}$ remains an unproven hypothesis.

In conclusion, we report that the MTRNR1 together with LHON primary mutation may complicate LHON phenotype sustaining the concept that the combinatorial effect of mitochondrial DNA mutations may have a synergistic role in worsening or widening the spectrum of LHON phenotype. In addition, we confirmed that increased mtDNA copy number is determinant in protecting from vision loss in presence of an LHON primary mutation.

\section{Additional files}

Additional file 1: Prioritization of mtDNA variants by MToolBox in LHON proband. All potentially deleterious mutations not contributing to the macro-haplogroup definition and, if non-synonymous, predicted as disease-associated by at least one of the pathogenicity prediction methods are reported as prioritized. $\mathbf{N t}$, nucleotide: $\mathbf{A A}$ amino acid dbSNP, single nucleotide polymorphism database. (DOC $110 \mathrm{~kb}$ )

Additional file 2: Fragment analysis report. Report of height and area of the peak signals representing the PCR fragments analyzed (base pairs; bp); length fragment species are expressed as arbitrary unit. (DOC 205 kb)

\section{Abbreviations}

BCVA: Best Corrected Visual Acuity; IQ: Intelligence Quotient; LE: Left eye; MTRNR1: Mitochondrial encoded 12S rRNA gene; OCT: Optical Coherent Tomography; RE: Right eye; RNFL: Retinal Nerve Fiber Layer Thickness

\section{Acknowledgments}

The Authors thank the family for participating. On behalf of all authors, the corresponding author states that there is no conflict of interest.

\section{Funding}

This work was supported by 2010 grants from the University of Bari (Fondi ex-60\%, 2009-2011) and Petruzzella00724113Prin - Finanziamento Università Prin 30 2009. The authors report no conflicts of interest. The authors alone are responsible for the content and writing.

Availability of data and materials

The data of the current study are available from the corresponding author on reasonable request.

\section{Authors' contributions}

$A B$ and VP gave substantial contributions to the conception and design of the work and to the acquisition and analysis of data as well as for drafting the work. LB; VG; PDB and AT, gave contributions to the design of the work and in the acquisition and analysis of data for the work. M-FDC.; SZ and SG made all the clinical examinations and gave contributions to the conception of the work. All the Authors gave final approval of the version to be published and agreement to be accountable for all aspects of the work in ensuring that questions related to the accuracy or integrity of any part of the work are appropriately investigated and resolved.

\section{Ethics approval and consent to participate}

Before recruitment into our study, all of the family members involved in the case report particularly, the parents of the patient have signed written informed consent for the molecular studies and publication of the medical data of themselves and their children. This consent was approved by the ethical committees of the Institutional Review Board of Azienda Consorziale Policlinico-Bari and University of Bari Aldo Moro.

\section{Consent for publication}

The patient's parents consented to the publication of the case and any accompanying medical data in writing.

\section{Competing interests}

The authors declare that they have no competing interests.

\section{Publisher's Note}

Springer Nature remains neutral with regard to jurisdictional claims in published maps and institutional affiliations.

\section{Author details}

${ }^{1}$ Dipartimento di Scienze Mediche di Base, Neuroscienze e Organi di Senso, Università degli Studi Aldo Moro, Piazza G. Cesare, 70124 Bari, Italy.

${ }^{2}$ Ospedale Casa Sollievo della Sofferenza IRCCS, UOC Genetica Medica, San Giovanni Rotondo, Italy. ${ }^{3}$ Istituto di Biomembrane, Bioenergetica e Biotecnologie Molecolari, IBIOM - CNR - Via G, Amendola 165/A, 70126 Bari, Italy. 


\section{Received: 9 February 2018 Accepted: 12 July 2018 Published online: 27 July 2018}

\section{References}

1. Yu-Wai-Man P, Griffiths PG, Chinnery PF. Mitochondrial optic neuropathies disease mechanisms and therapeutic strategies. Prog Retin Eye Res. 2011;30: $81-114$

2. Carelli V, Rugolo M, Sgarbi G, Ghelli A, Zanna C, Baracca A, et al. Bioenergetics shapes cellular death pathways in Leber's hereditary optic neuropathy: a model of mitochondrial neurodegeneration. Biochim Biophys Acta. 2004;1658:172-9.

3. Bi R, Zhang AM, Yu DD, Chen DN, Yao YG. Screening the three LHON primary mutations in the general Chinese population by using an optimized multiplex allele-specific PCR. Clin Chim Acta. 2010;411:1671-4.

4. Hudson G, Carelli V, Spruijt L, Gerards M, Mowbray C, Achilli A, et al. Clinical expression of Leber hereditary optic neuropathy is affected by the mitochondrial DNA-haplogroup background. Am J Hum Genet. 2007;81:228-33.

5. Nikoskelainen EK, Marttila RJ, Huoponen K, Juvonen V, Lamminen T, Sonninen $P$, et al. Leber's plus - neurological abnormalities in patients with Lebers hereditary optic neuropathy. J Neurol Neurosurg Psychiatry. 1995;59:160-4.

6. McFarland R, Chinnery PF, Blakely EL, Schaefer AM, Morris AAM, Foster SM, et al. Homoplasmy, heteroplasmy, and mitochondrial dystonia. Neurology. 2007;69:911-6

7. Meire FM, Vancoster R, Cochaux P, Obermaierkusser B, Candaele C, Martin JJ. Neurological disorders in members of families with Leber's hereditary optic neuropathy (Lhon) caused by different mitochondrial mutations. Ophthalmic Genet. 1995;16:119-26.

8. Watanabe M, Mita S, Takita T, Goto Y, Uchino M, Imamura S. Leber's hereditary optic neuropathy with dystonia in a Japanese family. J Neurol Sci. 2006:243:31-4.

9. Watanabe $Y$, Odaka M, Hirata K. Case of Leber's hereditary optic neuropathy with mitochondrial DNA 11778 mutation exhibiting cerebellar ataxia, dilated cardiomyopathy and peripheral neuropathy. J Neurol Sci. 2009;61:309-12.

10. Funakawa I, Kato H, Terao A, Ichihashi K, Kawashima S, Hayashi T, et al. Cerebellar-Ataxia in patients with Leber's hereditary optic neuropathy. J Neurol. 1995;242:75-7.

11. Niehusmann P, Surges R, von Wrede RD, Elger CE, Wellmer J, Reimann J, et al. Mitochondrial dysfunction due to Leber's hereditary optic neuropathy as a cause of visual loss during assessment for epilepsy surgery. Epilepsy Behav. 2011;20:38-43.

12. Grazina MM, Diogo LM, Garcia PC, Silva ED, Garcia TD, Robalo CB, et al. Atypical presentation of Leber's hereditary optic neuropathy associated to mtDNA 11778G >a point mutation-a case report. Eur J Paediatr Neurol. 2007; 11:115-8.

13. La Morgia C, Achilli A, lommarini L, Barboni P, Pala M, Olivieri A, et al. Rare $m t D N A$ variants in Leber hereditary optic neuropathy families with recurrence of myoclonus. Neurology. 2008;70:762-70.

14. Carelli V, Valentino ML, Liguori R, Meletti S, Vetrugno R, Provini F, et al. Leber's hereditary optic neuropathy (LHON/11778) with myoclonus: report of two cases. J Neurol Neurosurg Psychiatry. 2001;71:813-6.

15. Chinnery PF, Andrews RM, Turnbull DM, Howell N. Leber hereditary optic neuropathy: does heteroplasmy influence the inheritance and expression of the G11778A mitochondrial DNA mutation? Am J Med Genet. 2001;98:235-43.

16. Zeviani M, Di Donato S. Mitochondrial disorders. Brain. 2004;127:2153-72.

17. Y W-Man P, Turnbull DM, Chinnery PF. Leber hereditary optic neuropathy. J Med Genet 2002;39:162-9.

18. Giordano C, lommarini L, Giordano L, Maresca A, Pisano A, Valentino ML, et al. Efficient mitochondrial biogenesis drives incomplete penetrance in Leber's hereditary optic neuropathy. Brain. 2014;137:335-53.

19. Bianco A, Martinez-Romero I, Bisceglia L, D'Agruma L, Favia P, Ruiz-Pesini E, et al. Mitochondrial DNA copy number differentiates the Leber's hereditary optic neuropathy affected individuals from the unaffected mutation carriers. Brain. 2016;139:e1.

20. Bianco A, Bisceglia L, Russo L, Palese LL, D'Agruma L, Emperador S, et al. High mitochondrial DNA copy number is a protective factor from vision loss in Heteroplasmic Leber's hereditary optic neuropathy (LHON). Invest Ophthalmol Vis Sci. 2017;58:2193-7.

21. Bianco A, Bisceglia L, Russo L, D’Agruma L, Guerriero S, Petruzzella V. Leber's hereditary optic neuropathy (LHON) in an Apulian cohort of subjects. Acta Myol. 2017;36:163-77.
22. Mengel-From J, Thinggaard M, Dalgard C, Kyvik KO, Christensen K, Christiansen L. Mitochondrial DNA copy number in peripheral blood cells declines with age and is associated with general health among elderly. Hum Genet. 2014;133:1149-59.

23. Tommasi S, Favia $P$, Weigl $S$, et al. Mitochondrial DNA variants and risk of familial breast cancer: an exploratory study. Int J Oncol. 2014;44:1691-8.

24. Calabrese C, Simone D, Diroma MA, Santorsola M, Gutta C, Gasparre G, et al. MToolBox: a highly automated pipeline for heteroplasmy annotation and prioritization analysis of human mitochondrial variants in high-throughput sequencing. Bioinformatics. 2014;30:3115-7.

25. ViennaRNA Web Services (http://rna.tbi.univie.ac.at/cgi-bin/RNAWebSuite/ RNAfold.cgi).

26. Chinnery PF, Thorburn DR, Samuels DC, White SL, Dahl HM, Turnbull DM, et al. The inheritance of mitochondrial DNA heteroplasmy: random drift, selection or both? Trends Genet. 2000;16:500-5.

27. Giordano L, Deceglie S, d'Adamo P, Valentino M, La Morgia C, Fracasso F, et al. Cigarette toxicity triggers Leber's hereditary optic neuropathy by affecting mtDNA copy number, oxidative phosphorylation and ROS detoxification pathways. Cell Death Dis. 2015;6

28. Kwittken J, Barest HD. The neuropathology of hereditary optic atrophy (Leber's disease); the first complete anatomic study. Am J Pathol. 1958;34: 185-207.

29. Finsterer J, Bastovansky A. Multiorgan disorder syndrome (MODS) in an octagenarian suggests mitochondrial disorder. Rev Med Chil. 2015;143:1210-4.

30. Rudenskaia GE, Zakharova E, Adarcheva LS, Mikhailova EN, Karlova IZ. Leber's hereditary optic atrophy: neurological and other non-optic appearances. Zh Nevrol Psikhiatr Im S S Korsakova. 2004;104:38-42.

31. Saccone C, De Giorgi C, Gissi C, Pesole G, Reyes A. Evolutionary genomics in Metazoa: the mitochondrial DNA as a model system. Gene. 1999;238:195-209.

32. Bendall KE, Sykes BC. Length heteroplasmy in the first hypervariable segment of the human mtDNA control region. Am J Hum Genet. 1995;57:248-56.

33. Li RH, Xing GQ, Yan M, Cao X, Liu XZ, Bu XK, Guan MX. Cosegregation of Cinsertion at position 961 with the A1555G mutation of the mitochondrial $12 S$ rRNA gene in a large chinese family with maternally inherited hearing loss. Am J Med Genet A. 2004;124A:113-7.

34. Epshtein V, Cardinale CJ, Ruckenstein AE, Borukhov S, Nudler E. An allosteric path to transcription termination. Mol Cell. 2007;28:991-1001.

35. Prezant TR, Agapian JV, Bohlman MC, Bu XD, Oztas S, Qiu WQ, et al. Mitochondrial ribosomal-Rna mutation associated with both antibioticinduced and non-Syndromic deafness. Nat Genet. 1993;4:289-94.

36. Zhao H, Li RH, Wang QJ, Yan QF, Deng JH, Han DY et al. Maternally inherited aminoglycoside-induced and nonsyndromic deafness is associated with the novel C1494T mutation in the mitochondrial 12S rRNA gene in a large chinese family. Am J Hum Genet 2004;74:139-152.

37. Lu J, Li Z, Zhu Y, Yang A, Li R, Zheng J, et al. Mitochondrial 12S rRNA variants in 1642 Han Chinese pediatric subjects with aminoglycosideinduced and nonsyndromic hearing loss. Mitochondrion. 2010;10:380-90.

38. Kokotas H, Petersen MB, Willems PJ. Mitochondrial deafness. Clin Genet. 2007:71:379-91.

39. Rydzanicz M, Wrobel M, Pollak A, Gawecki W, Brauze D, KostrzewskaPoczekaj M, Wojsyk-Banaszak I, et al. Mutation analysis of mitochondrial 125 rRNA gene in polish patients with non-syndromic and aminoglycosideinduced hearing loss. Biochem Biophys Res Commun. 2010;395:116-21.

40. Guan MX. Mitochondrial 12S rRNA mutations associated with aminoglycoside ototoxicity. Mitochondrion. 2011;11:237-45.

41. Neefs JM, Van de Peer Y, De Rijk P, Goris A, De Wachter R. Compilation of small ribosomal subunit RNA sequences. Nucleic Acids Res. 1991;19(Suppl): 1987-2015.

42. Li Z, Li R, Chen J, Liao Z, Zhu Y, Qian Y, et al. Mutational analysis of the mitochondrial $12 S$ rRNA gene in Chinese pediatric subjects with aminoglycoside-induced and non-syndromic hearing loss. Hum Genet. 2005;117:9-15.

43. Khan MA, Khan S, Windpassinger C, Badar M, Nawaz Z, Mohammad RM. The molecular genetics of autosomal recessive Nonsyndromic intellectual disability: a mutational continuum and future recommendations. Ann Hum Genet. 2016;80:342-68.

44. Wang J, Lin Z, Liu L, Xu HQ, Shi YW, Yi YH, et al. Epilepsy-associated genes. Seizure. 2017:44:11-20. 